\title{
Core excitations across the neutron shell gap in ${ }^{207} \mathrm{Tl}$
}

\author{
E. Wilson ${ }^{\mathrm{a}, *, 1}$, Zs. Podolyák ${ }^{\mathrm{a}}$, H. Grawe ${ }^{\mathrm{b}}$, B.A. Brown ${ }^{\mathrm{c}}$, C.J. Chiara ${ }^{\mathrm{d}, \mathrm{e}, 2}{ }^{2}, \mathrm{~S}_{\text {. Zhu }}{ }^{\mathrm{e}}$, B. Fornal $^{\mathrm{f}}$, \\ R.V.F. Janssens ${ }^{e}$, C.M. Shand ${ }^{a}$, M. Bowry ${ }^{a, 3}$, M. Bunce ${ }^{a}$, M.P. Carpenter ${ }^{\mathrm{e}}$, \\ N. Cieplicka-Oryńczak ${ }^{\text {f,4 }}$, A.Y. Deo ${ }^{\text {g,5 }}{ }^{\text {, G.D. Dracoulis }}{ }^{\text {h }}$, C.R. Hoffman ${ }^{\text {e }}$, R.S. Kempley ${ }^{\mathrm{a}}$, \\ F.G. Kondev ${ }^{\text {i }}$, G.J. Lane ${ }^{\text {h, T. Lauritsen }}{ }^{\text {e }}$, G. Lotay ${ }^{a, j}$, M.W. Reed ${ }^{a, 6}$, P.H. Regan ${ }^{a, j}$, \\ C. Rodríguez Triguero ${ }^{k}$, D. Seweryniak ${ }^{e}$, B. Szpak ${ }^{f}$, P.M. Walker ${ }^{a}$ \\ a Department of Physics, University of Surrey, Guildford, GU2 7XH, UK \\ b GSI Helmholtzzentrum für Schwerionenforschung GmbH, D-64291 Darmstadt, Germany \\ c Department of Physics and Astronomy and National Superconducting Cyclotron Laboratory, Michigan State University, East Lansing, MI 48824-1321, USA \\ d Department of Chemistry and Biochemistry, University of Maryland, College Park, MD 20742, USA \\ e Physics Division, Argonne National Laboratory, Argonne, IL 60439, USA \\ ${ }^{\mathrm{f}}$ H. Niewodniczański Institute of Nuclear Physics, PL-31342 Kraków, Poland \\ ${ }^{g}$ Department of Physics, University of Massachusetts Lowell, Lowell, MA 01854, USA \\ h Department of Nuclear Physics, Research School of Physics and Engineering, Australian National University, Canberra, Australian Capital Territory 0200, \\ Australia

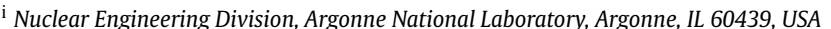 \\ j National Physical Laboratory, Hampton Road, Teddington, Middlesex, TW11 OLW, UK \\ ${ }^{\mathrm{k}}$ School of Computing, Engineering and Mathematics, University of Brighton, Brighton, BN2 4GL, UK
}

\section{A R T I C L E I N F O}

\section{Article history:}

Received 4 February 2015

Accepted 23 April 2015

Available online 5 May 2015

Editor: D.F. Geesaman

\begin{abstract}
A B S T R A C T
The single closed-neutron-shell, one proton-hole nucleus ${ }^{207} \mathrm{Tl}$ was populated in deep-inelastic collisions of a ${ }^{208} \mathrm{~Pb}$ beam with a ${ }^{208} \mathrm{~Pb}$ target. The yrast and near-yrast level scheme has been established up to high excitation energy, comprising an octupole phonon state and a large number of core excited states. Based on shell-model calculations, all observed single core excitations were established to arise from the breaking of the $N=126$ neutron core. While the shell-model calculations correctly predict the ordering of these states, their energies are compressed at high spins. It is concluded that this compression is an intrinsic feature of shell-model calculations using two-body matrix elements developed for the description of two-body states, and that multiple core excitations need to be considered in order to accurately calculate the energy spacings of the predominantly three-quasiparticle states.
\end{abstract}

(C) 2015 The Authors. Published by Elsevier B.V. This is an open access article under the CC BY license (http://creativecommons.org/licenses/by/4.0/). Funded by SCOAP ${ }^{3}$.
The shell model is a universal approach for the description of microscopic multi-particle systems, used successfully for such

\footnotetext{
* Corresponding author.

E-mail address: ewilson@richmond.edu (E. Wilson).

1 Present address: Department of Physics, University of Richmond, Richmond, VA 23173, USA.

2 Present address: U.S. Army Research Laboratory, Adelphi, MD 20783, USA.

3 Present address: Department of Physics and Astronomy and National Superconducting Cyclotron Laboratory, Michigan State University, East Lansing, MI 48824-1321, USA.

4 Present address: Università degli Studi di Milano, 20133 Milan, Italy.

5 Present address: Department of Physics, Indian Institute of Technology Roorkee, Roorkee, 247667, India.

6 Present address: Department of Nuclear Physics, Research School of Physics and Engineering, Australian National University, Canberra, Australian Capital Territory 0200, Australia.
}

diverse structures as nuclei, electrons in an atom or metallic clusters [1-3]. In the case of nuclei, the shell model works well in explaining the structures based on valence nucleons in the vicinity of doubly-magic nuclei. Far from the magic nuclei, where a large number of valence nucleons are present, shell-model calculations become computationally difficult, and other types of models, collective models, are used more widely. In nuclei with a moderate number of valence nucleons, the study of the interplay between single-particle and collective structure is of high interest.

Excited states in doubly-magic nuclei are the result of breaking the neutron or proton core. The treatment of such states in the shell model is more difficult as orbitals from several different major shells and their interactions have to be considered. The present work addresses the region around the heaviest known doubly-magic nucleus; ${ }^{208} \mathrm{~Pb}$. This nucleus has the peculiarity that 
its first excited state is a collective $3^{-}$excitation, with a highly mixed and complex wave function. The higher lying states have much simpler wave functions, dominated by well defined particlehole configurations. Low energy states in nuclei with one single valence particle/hole outside ${ }^{208} \mathrm{~Pb}$ are characterised by singleparticle structures, while higher energy states are the result of core breaking. Therefore, they provide an ideal testing ground for how collectivity arises from single-particle structures.

Deep-inelastic reactions involving a ${ }^{208} \mathrm{~Pb}$ target or beam are effective in populating nuclei in the vicinity of ${ }^{208} \mathrm{~Pb}$. They were used to study yrast and close to yrast states, for example, in ${ }^{206} \mathrm{Hg}$ [4]. They are also ideally suited to study high-spin states [5]. In this letter we report results on the singly magic ${ }^{207} \mathrm{Tl}_{126}$ nucleus, obtained from deep-inelastic reactions.

Nuclei were synthesised in the collisions of a ${ }^{208} \mathrm{~Pb}$ beam and a ${ }^{208} \mathrm{~Pb}$ target, using the ATLAS accelerator at Argonne National Laboratory. Therefore, the nucleus of interest, ${ }^{207} \mathrm{Tl}$, was populated both as a beam-like and as a target-like reaction product. The beam had an energy of $1446 \mathrm{MeV}, 20 \%$ above the Coulomb barrier, and all reaction products were stopped in the $75 \mathrm{mg} / \mathrm{cm}^{2}$-thick target. The beam current was $\sim 0.25$ particle-nA on average, and the experiment ran for $\sim 7$ days. The beam possessed a natural frequency such that a pulse occurred once every 82.5 ns. Four of every five pulses were deflected, leading to a beam-off period of 412.5 ns. Consequently, the experiment was sensitive to both prompt and isomeric decays, the latter having a half-life of up to a microsecond. The $\gamma$ rays were detected with the Gammasphere array [6,7], consisting of 101 HPGe detectors and their BGO anti-Compton shields. The Gammasphere array was energy and efficiency calibrated using standard $\gamma$-ray sources. Tantalum, cadmium and copper absorbers were positioned in front of the detectors in order to reduce the dominant $\mathrm{Pb}$ X-ray yields.

The pulse-processing trigger required three coincident $\gamma$-rays detected within $2 \mu$ s of each other. Further details on the experimental conditions have been given in our previous conference papers [8,9], and in Ref. [10].

The data were sorted into three-dimensional histograms of coincident $\gamma$-ray energies, or $\gamma \gamma \gamma$ cubes, with different time conditions. A combination of prompt and delayed cubes were created. In ${ }^{207} \mathrm{Tl}$, we found no evidence of delayed transitions in the present range of sensitivity, therefore all results presented here are from the prompt $\gamma$-ray data. In addition, the data were sorted in twodimensional $\gamma \gamma$ matrices for angular correlation and angular distribution studies. The angular distribution matrices were produced with $\gamma$ rays from individual angles on one axis, with all angles on the other one.

According to the 2011 Nuclear Data Sheets evaluations [11], the highest state with a confirmed spin in ${ }^{207} \mathrm{Tl}$ is a $11 / 2^{-}$level, which is interpreted as the single-hole $\pi h_{11 / 2}^{-1}$ state. Several transitions observed in a ${ }^{136} \mathrm{Xe}+{ }^{208} \mathrm{~Pb}$ deep-inelastic reaction had been identified as populating this state. Among these, one with an energy of $2464.9 \mathrm{keV}$ was interpreted as a stretched-E3 transition deexciting the $17 / 2^{+}$state [12]. The low-energy structure of ${ }^{207} \mathrm{Tl}$, with closed $N=126$ neutron-shell and one proton-hole outside the $Z=82$ magic number, is dominated by single proton-hole states. The ground-state has a $\pi s_{1 / 2}^{-1}$ configuration, followed by excited states of $\pi d_{3 / 2}^{-1}, \pi h_{11 / 2}^{-1}$, and $\pi d_{5 / 2}^{-1}$ character. The $11 / 2^{-}$state is isomeric with a half-life of $1.33 \mathrm{~s}$ [13]. All the $\gamma$ rays observed in the present work populate this long-lived excitation, directly or indirectly.

In the deep-inelastic collision process, the partner nucleus of ${ }^{207} \mathrm{Tl}$ is ${ }^{209} \mathrm{Bi}$, with a well known yrast level scheme [14]. By selecting low-lying transitions in ${ }^{209} \mathrm{Bi}$, we observe both ${ }^{209} \mathrm{Bi}$ and ${ }^{207} \mathrm{Tl}$ transitions in coincidence with these, as illustrated in Fig. 1a. Some
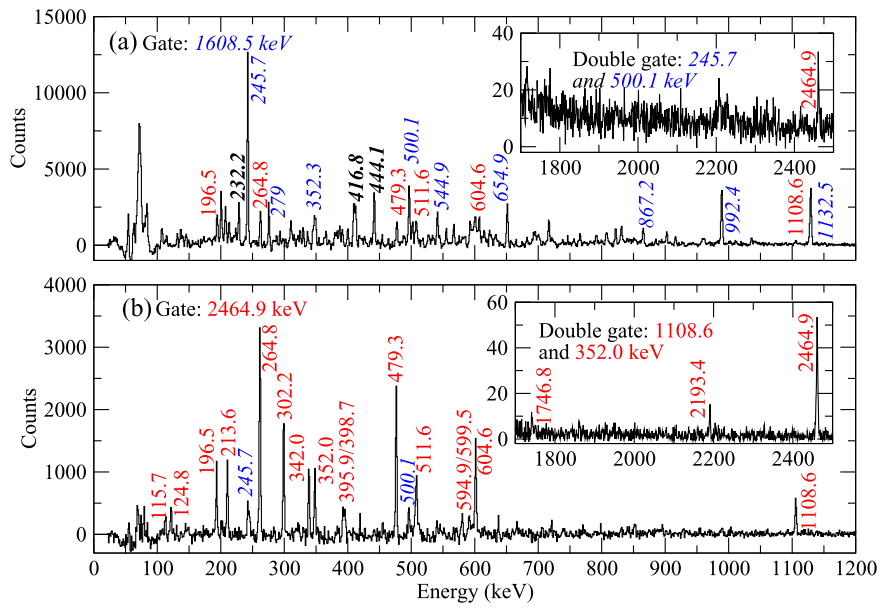

Fig. 1. (Colour online.) (a) $\gamma$-ray transitions in coincidence with the $1608.5-\mathrm{keV}$ transition from ${ }^{209} \mathrm{Bi}$. Gamma lines from reaction partners ${ }^{207} \mathrm{Tl}$ and ${ }^{209} \mathrm{Bi}$ are visible. (b) Spectrum obtained by gating on the $2464.9-\mathrm{keV}$ transition from ${ }^{207} \mathrm{Tl}$. The transitions labelled in red are assigned to ${ }^{207} \mathrm{Tl}$, while those labelled in blue and italics are from ${ }^{209} \mathrm{Bi}$. The transitions labelled with bold are from ${ }^{208} \mathrm{~Pb}$. The insets show the first and second core-breaking transitions in ${ }^{207} \mathrm{Tl}$.
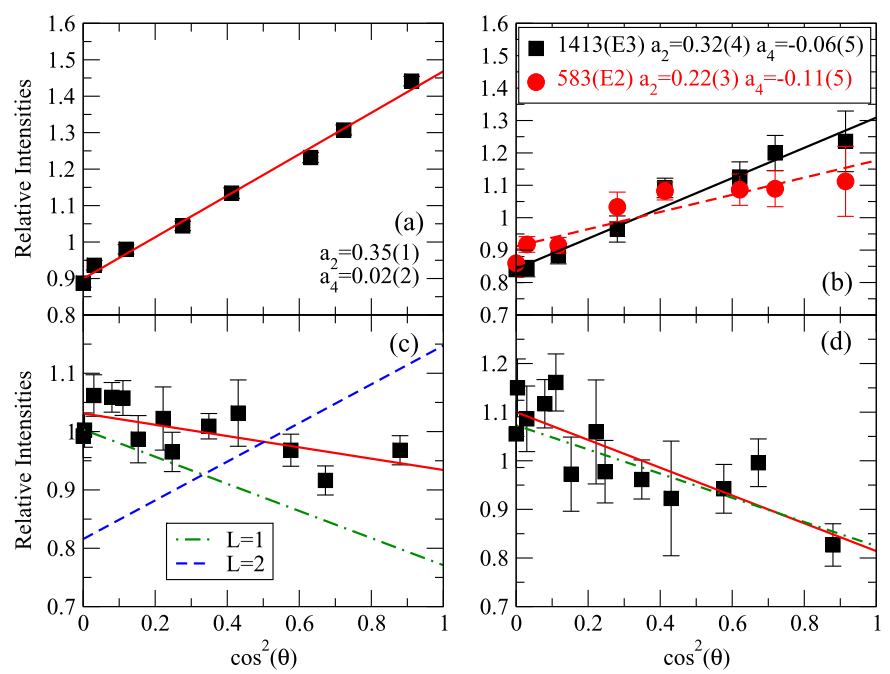

Fig. 2. (a) Angular distribution of the 2464.9-keV E3 transition, obtained from ungated spectra. (b) Comparison of angular distributions of known E2 and E3 transitions from ${ }^{208} \mathrm{~Pb}$, gated on the $2614.5-\mathrm{keV}$ transition. Panels (c) and (d) are the angular correlations obtained for the 264.8-keV M1 + E2 and 302.2-keV E1 transitions, respectively, gated on the $2464.9-\mathrm{keV}$ E3 transition. $L=1,2$ lines are the theoretical angular correlation patterns for the transitions; $L$ is the angular momentum of the stretched $\gamma$ ray.

of these were previously identified by Rejmund et al. [11,12]. By gating on the high-energy 2464.9-keV $\gamma$-ray transition, we identify a large number of transitions belonging to ${ }^{207} \mathrm{Tl}$ (see Fig. $1 \mathrm{~b}$ ). Double coincidence gates on these were used to build the level scheme of ${ }^{207} \mathrm{Tl}$. As deep-inelastic reactions are known to populate predominantly yrast and close to yrast states, we expect that the spins increase with excitation energy. Spins and parities were assigned based on decay patterns as well as angular correlation [15] and angular distribution analyses of the $\gamma$ rays. The 2464.9-keV line is the strongest transition, and the only one clearly visible in the total projection of the $\gamma \gamma \gamma$ coincidence cube. The angular distribution analysis clearly demonstrates its octupole character, as shown in Fig. 2. The $a_{2}$ and $a_{4}$ coefficients are very similar to those of E3 transitions in other nuclei $[10,16]$. Additional examples of angular correlation and distribution measurements are also 
Table 1

$\gamma$-ray transitions associated with ${ }^{207} \mathrm{Tl}$ observed in the present experiment; $\gamma$-ray energies, relative intensities from the current work as well as from literature [11,12], $a_{2}$ and $a_{4}$ coefficients from angular correlations, as well as the deduced character of the transitions are given. The angular correlations are with respect to the 2464.9-keV E3 transition. The transitions marked with a '*' are from states with excitation energy over $7 \mathrm{MeV}$ and they are not placed in the level scheme. Many of the transitions with $E_{X} \geq 7 \mathrm{MeV}$ are expected to be from core-breaking states.

\begin{tabular}{clllrl}
\hline$E_{\gamma}(\mathrm{keV})$ & $I_{\gamma}$ & $I_{\gamma}[11,12]$ & \multicolumn{1}{l}{$a_{2}$} & \multicolumn{1}{l}{$a_{4}$} & $\sigma L$ \\
\hline 115.7 & $11(3)$ & & & & \\
124.8 & $14(3)$ & & & & \\
129.3 & $3(2)$ & & & & \\
196.5 & $23(3)$ & & $-0.11(5)$ & $0.05(6)$ & $\mathrm{M} 1$ \\
213.6 & $24(3)$ & & $-0.21(4)$ & $-0.02(6)$ & $\mathrm{M} 1+\mathrm{E} 2$ \\
264.8 & $100(10)$ & $58(7)$ & $-0.07(2)$ & $0.03(3)$ & $\mathrm{M} 1+\mathrm{E} 2$ \\
302.2 & $40(4)$ & $40(6)$ & $-0.17(4)$ & $-0.00(5)$ & $\mathrm{E} 1$ \\
342.0 & $42(3)$ & $26(5)$ & $-0.04(3)$ & $-0.05(4)$ & $\mathrm{M} 1$ \\
352.0 & $23(5)$ & & $0.03(4)$ & $-0.09(6)$ & $\mathrm{E} 2$ \\
$395.9^{*}$ & $21(3)$ & & & & \\
$398.7^{*}$ & $12(3)$ & & & & \\
$422.5^{*}$ & $5(3)$ & & & & \\
479.3 & $59(6)$ & $100(10)$ & $0.26(4)$ & $0.07(5)$ & $\mathrm{M} 1+\mathrm{E} 2$ \\
511.6 & $28(3)$ & $30(5)$ & & & \\
$594.9^{*}$ & $10(3)$ & & & & \\
$599.5^{*}$ & $7(4)$ & & & & \\
604.6 & $51(5)$ & $53(7)$ & $0.09(4)$ & $0.02(5)$ & $\mathrm{E} 2$ \\
1108.6 & $31(3)$ & $19(5)$ & $-0.09(6)$ & $-0.01(10)$ & $\mathrm{M} 1 / \mathrm{E} 1$ \\
$1746.8^{*}$ & $5(2)$ & & & & \\
$2193.4^{*}$ & $4(2)$ & & & & \\
2464.9 & & & & &
\end{tabular}

shown on the same figure. The $\gamma$-ray intensities as well as the results of the angular correlation analysis are given in Table 1. Intensity balance analysis shows that the $264.8 \mathrm{keV} \gamma$ ray has mixed M1 + E2 character, in agreement with the angular correlation/distribution results. The experimentally deduced level scheme is presented in Fig. 3. This level scheme is considered to be reliable up to a $6985-\mathrm{keV}$ excitation energy and spin $35 / 2$. The parity of this state is tentatively assigned to be positive, due to comparisons with shell-model calculations. Several higher-lying $\gamma$ rays were also observed, but their position in the level scheme is uncertain and they are not shown in Fig. 3. The high-energy transitions breaking the core, the $2464.9-\mathrm{keV}$ E3 as well as the second core breaking 1746.8- and 2193.4-keV $\gamma$ rays are seen in the inset of Fig. $1 \mathrm{~b}$.

In order to a have better understanding of the structure of ${ }^{207} \mathrm{Tl}$, shell-model calculations have been performed. Two different sets of interactions and model spaces were considered. The OXBASH code [17] was employed. The single-particle/hole energies relative to ${ }^{208} \mathrm{~Pb}$ were taken from experimental values. Calculations were performed for ${ }^{208} \mathrm{~Pb}$ and for the four neighbouring singleparticle/hole nuclei ${ }^{207} \mathrm{Tl},{ }^{209} \mathrm{Bi},{ }^{207,209} \mathrm{~Pb}$. The details of the two shell-model calculations are as follows:

(1) KHH7B interaction: The model space considered consisted of the proton orbitals $d_{5 / 2}, h_{11 / 2}, d_{3 / 2}, s_{1 / 2}$ below $Z=82$ and the $h_{9 / 2}, f_{7 / 2}, i_{13 / 2}$ ones above it, and the neutron orbitals $i_{13 / 2}, p_{3 / 2}$, $f_{5 / 2}, p_{1 / 2}$ below $N=126$ and $g_{9 / 2}, i_{11 / 2}, j_{15 / 2}$ above. The cross shell two-body interaction matrix elements (TBMEs) are based on the H7B G-matrix [18], while the neutron-proton TBMEs are based on the Kuo-Herling interaction [19] as modified in [20]. These calculations describe accurately valence particle excitations (when no core-breaking is needed). They were used extensively on nuclei below $Z=82$ along the $N=126$ line [21-24], as well as for both in the $N>126$ [25] and $N<126$ [5,24] regions.

(2) KHM3Y interaction: The model space consisted of the proton orbitals $\mathbf{g}_{7 / 2}, d_{5 / 2}, h_{11 / 2}, d_{3 / 2}, s_{1 / 2}$ below $Z=82$ and $h_{9 / 2}, f_{7 / 2}$, $i_{13 / 2}, \mathbf{f}_{\mathbf{5} / \mathbf{2}}, \mathbf{p}_{\mathbf{3} / \mathbf{2}}, \mathbf{p}_{\mathbf{1} / \mathbf{2}}$ above it, and the neutron orbitals $i_{13 / 2}, p_{3 / 2}$, $f_{5 / 2}, p_{1 / 2}, \mathbf{h}_{\mathbf{9} / \mathbf{2}}, \mathbf{f}_{7 / 2}$ below $N=126$ and $g_{9 / 2}, i_{11 / 2}, j_{15 / 2}, \mathbf{g}_{7 / 2}$, $\mathbf{d}_{\mathbf{5} / \mathbf{2}}, \mathbf{d}_{\mathbf{3} / \mathbf{2}}, \mathbf{s}_{\mathbf{1} / \mathbf{2}}$ above. The additional orbitals, compared to the
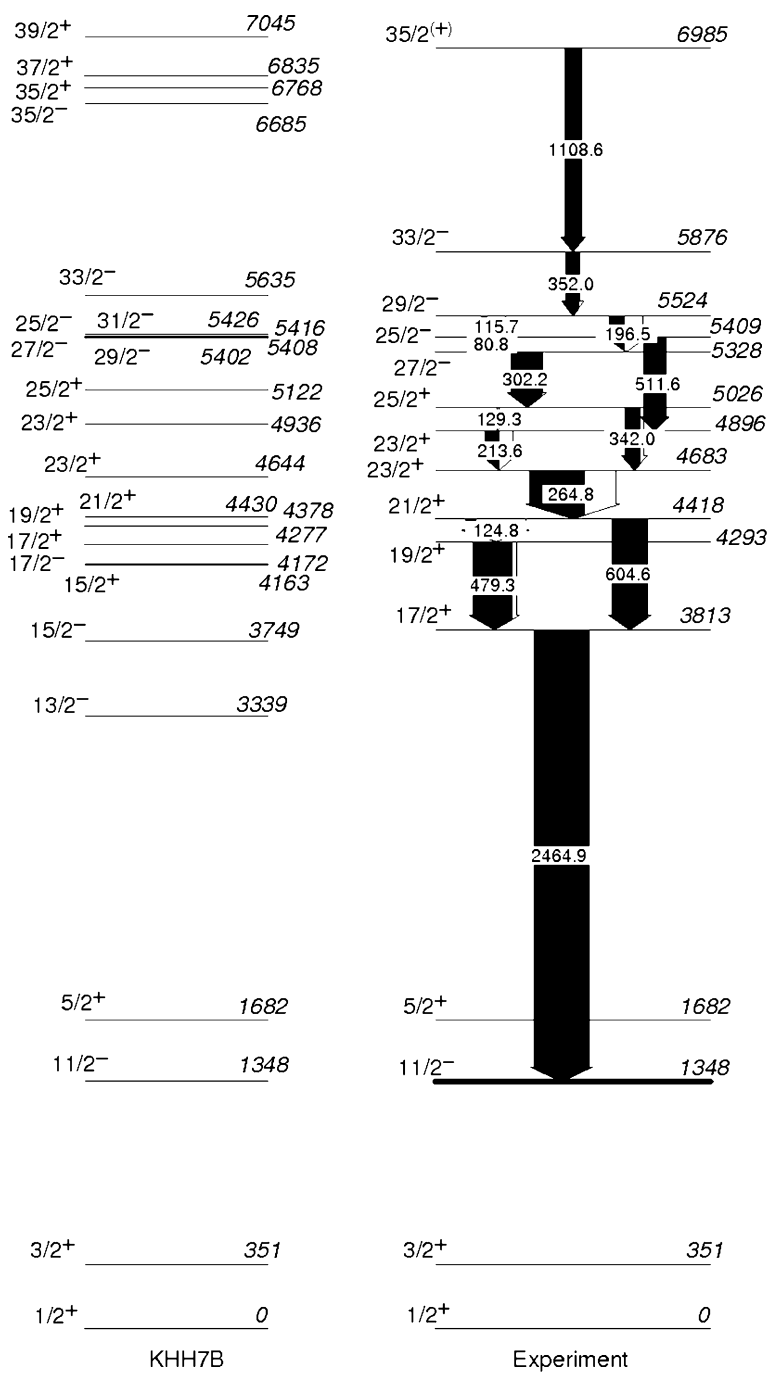

Fig. 3. Comparison between the experimental and the KHH7B shell-model level schemes of ${ }^{207} \mathrm{Tl}$. See the text for details.

KHH7B calculations, are shown in bold. The cross-shell, two-body matrix elements are based on the M3Y interaction [26], while the neutron-proton interactions are based on the Kuo-Herling interaction [19] as modified in Ref. [20]. Such calculations gave a good description of both valence particle excitations around ${ }^{208} \mathrm{~Pb}$ [20] and of single- and double-octupole states in ${ }^{208} \mathrm{~Pb}$ [27].

In the present calculations core excitations across the ${ }^{208} \mathrm{~Pb}$ double-shell closure were allowed. The calculations were done with truncation to one-particle one-hole $(1 p 1 h)$ excitations for protons and neutrons. Mixing between $t=0$ valence and coreexcited configurations was blocked, as experimental binding energies and single-particle/hole excitation energies (SPE/SHE) were used as model input data and, therefore, do not need further correlations. First, we compared the calculations with experimental data in the case of the core ${ }^{208} \mathrm{~Pb}$ nucleus. There is good agreement between shell-model and experiment in the case of core excited states, as shown in Fig. 4. The real difference between the two calculations is on the $3^{-}$octupole state. The KHH7B Hamiltonian considerably overestimates its energy, while the KHM3Y calculation slightly underestimates it. This state is collective in nature, therefore its wave function is complex containing a large number of core excitations. The difference between the two calculations regarding the octupole state is well understood. It is related to the 


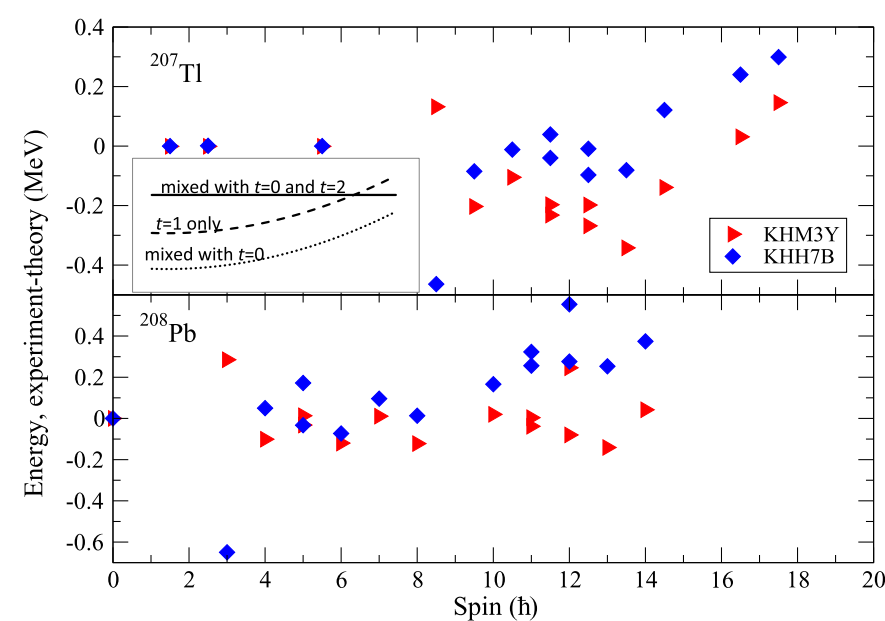

Fig. 4. Energy differences between experimental and shell-model excitation energies in ${ }^{208} \mathrm{~Pb}$ and ${ }^{207} \mathrm{Tl}$. The sketch in the inset shows the effect on $t=1$ states of mixing with $t=0$ and $t=2$ states. See the text for a detailed discussion.

Table 2

Configurations of states in ${ }^{207} \mathrm{Tl}$, from the KHH7B shell-model calculations. Only the main components, with weight $>20 \%$, are given.

\begin{tabular}{rrlll}
\hline $\begin{array}{l}E_{X}(\mathrm{exp}) \\
\mathrm{keV}\end{array}$ & $\begin{array}{l}E_{X}(\text { the }) \\
\mathrm{keV}\end{array}$ & $J^{\pi}$ & Configuration & $\begin{array}{l}\text { Partition } \\
\%\end{array}$ \\
\hline 0 & 0 & $1 / 2^{+}$ & $\pi s_{1 / 2}^{-1}$ & 100 \\
351 & 351 & $3 / 2^{+}$ & $\pi d_{3 / 2}^{-1}$ & 100 \\
1348 & 1348 & $11 / 2^{-}$ & $\pi h_{11 / 2}^{-1}$ & 100 \\
1682 & 1682 & $5 / 2^{+}$ & $\pi d_{5 / 2}^{-1}$ & 100 \\
3813 & 4277 & $17 / 2^{+}$ & $\pi h_{11 / 2}^{-1} \nu p_{1 / 2}^{-1} g_{9 / 2}$ & 63 \\
4293 & 4378 & $19 / 2^{+}$ & $\pi h_{11 / 2}^{-1} \nu p_{1 / 2}^{-1} g_{9 / 2}$ & 59 \\
4418 & 4430 & $21 / 2^{+}$ & $\pi s_{1 / 2}^{-1} \nu i_{13 / 2}^{-1} g_{9 / 2}$ & 71 \\
4683 & 4644 & $23 / 2^{+}$ & $\pi s_{1 / 2}^{-1} \nu i_{13 / 2}^{-1} g_{9 / 2}$ & 81 \\
4896 & 4936 & $23 / 2^{+}$ & $\pi d_{3 / 2}^{-1} \nu i_{13 / 2}^{-1} g_{9 / 2}$ & 39 \\
& & & $\pi h_{11 / 2}^{-1} \nu f_{5 / 2}^{-1} g_{9 / 2}$ & 30 \\
5026 & 5122 & $25 / 2^{+}$ & $\pi h_{11 / 2}^{-1} \nu f_{5 / 2}^{-1} g_{9 / 2}$ & 79 \\
5524 & 5402 & $29 / 2^{-}$ & $\pi h_{11 / 2}^{-1} \nu i_{13 / 2}^{-1} g_{9 / 2}$ & 94 \\
5328 & 5408 & $27 / 2^{-}$ & $\pi h_{11 / 2}^{-1} \nu i_{13 / 2}^{-1} g_{9 / 2}$ & 93 \\
5876 & 5635 & $33 / 2^{-}$ & $\pi h_{11 / 2}^{-1} \nu i_{13 / 2}^{-1} g_{9 / 2}$ & 95 \\
6985 & 6768 & $35 / 2^{+}$ & $\pi h_{11 / 2}^{-1} \nu i_{13 / 2}^{-1} j_{15 / 2}$ & 94 \\
$(6985)$ & 6685 & $35 / 2^{-}$ & $\pi h_{11 / 2}^{-1} \nu i_{13 / 2}^{-1} i_{11 / 2}$ & 94 \\
\hline
\end{tabular}

number of orbitals considered, and especially to the $\Delta j=\Delta l=3$ pairs, as explained in detail in [16].

Next, we compared the shell-model calculations with the experimental excitation energies in the case of ${ }^{207} \mathrm{Tl}$ (see Fig. 4). Again, there is a good agreement in the case of the core excited states. Similarly to ${ }^{208} \mathrm{~Pb}$, the energy of the $17 / 2^{+}$state with $\pi h_{11 / 2}^{-1}$ coupled to the collective $3^{-}$octupole phonon is overestimated by the KHH7B shell model and underestimated by the KHM3Y one [16]. The KHH7B shell-model level scheme is shown next to the experimental one in Fig. 3. There is a direct correspondence for all experimental levels and the branching ratios are reasonably reproduced as well [10]. The most intense experimental transitions correspond with those predicted by theory, with the exception of the transition populating the $23 / 2_{1}^{+}$and $23 / 2_{2}^{+}$states. The leading configurations are given in Table 2 . All states can be understood as the result of the breaking of the $N=126$ neutron core. With the exception of the yrast $21 / 2^{+}$and $23 / 2^{+}$states, all other coreexcited states are built on the high spin proton orbital $h_{11 / 2}$.
A closer inspection of Fig. 4 points to a systematic effect for states in ${ }^{207} \mathrm{Tl}$ with spins above $29 / 2 \hbar$. The difference between experimental and theoretical excitation energies increases with spin. In other words, the calculated levels are compressed at high-spin (see Fig. 3). This observation holds for both shell-model calculations. Here, a qualitative explanation of this behaviour is presented.

Mixing between states with different numbers of core excitations $(t=0,1,2 \ldots)$ modifies their relative energies. In order to estimate how much the inclusion of additional excitations affects a state, the single-particle states were examined in the four nuclei around ${ }^{208} \mathrm{~Pb}$. The mixing of $t=0$ (no core excitations) and the $t=1$ (core excitations) states, pushed down the energies of the $t=0$ states by $\sim 250 \mathrm{keV}$. The energy change is correlated with the number of excitations induced, and it is smaller at lower spins. At the same time, the energies of $t=1$ states in the spin region of interest, $I>11 / 2 \hbar$, are not affected since there are no such $t=0$ states to mix with (see inset of Fig. 4). In the case of the ground state in ${ }^{207} \mathrm{Tl}$, the energy change was $281 \mathrm{keV}$.

Inclusion of $t=2$ states in the calculations will push the $t=1$ states down in energy. The energy shift of the $t=1$ states will be correlated with the number of available $t=2$ states with given spin-parity. The number of high-spin states is lower than the lowto-medium spin states. Consequently, the high-spin $t=1$ states will be pushed down less by this mixing with $t=2$ states. Therefore, the compression of the high-spin states in the $t=1$ shellmodel calculations can be understood; the $t=2$ excitations have a spin-parity distribution, and comparatively few of them will have high, $I>15 \hbar$, spin. The effect of mixing on the $t=1$ core-excited states is sketched in the inset of Fig. 4. While mixing with $t=2$ states might be able to solve the problem of the compression of high-spin states, it would not result in correct excitation energies. Mixing with $t=2$ states will affect the energies of the singleparticle states as well, including the ground state. As these spins can be produced with relatively low energy by lifting an $I=0$ pair above the gap, the effect is larger than on the $t=1$ levels. So, in reality, including at least $t=3$ states is needed in order to get the correct excitation energies. However, due to computational limitations, calculations with three (or five) particle-hole excitations cannot be performed for ${ }^{207} \mathrm{Tl}$. It should be noted that, in the present case, where the single-particle energies are taken from experiment, mixing of states with different $t$ values will result in the wrong computed mass.

A similar discrepancy between experiment and theory when only one core excitation was considered was previously noted in the ${ }^{100} \mathrm{Sn}$ region [28]. In order to achieve a good description of the excitation energies of core-excited states in ${ }^{98} \mathrm{Cd}$, several, $t=5$, particle-hole excitations across the closed $Z=N=50$ shell had to be accounted for. At lower masses, such calculations can be carried out due to the lower number of available orbitals within the shells. Around ${ }^{100} \mathrm{Sn}$, only a single orbital, the $g_{9 / 2}$ one, needs to be considered below $Z=N=50$, with $g_{7 / 2}, d_{5 / 2}$, and $h_{11 / 2}$ states above the shell gaps. On the other hand, around ${ }^{208} \mathrm{~Pb}$, there are a large number of orbitals both below and above the proton and neutron shell gaps to be taken into account.

Due to computational challenges (large number of orbitals from four major shells have to be considered), the shell-model framework with realistic interactions is rarely used to study core-excited states around ${ }^{208} \mathrm{~Pb}[27,29]$. The majority of the works involved effective residual interactions, which were derived from experiment. In this case, the octupole state is treated as an independent excitation [30]. These calculations have high predictive power [31,32], however, they provide less insight into the microscopic structure of the excitations. 
In conclusion, ${ }^{207} \mathrm{Tl}_{126}$ was populated in a ${ }^{208} \mathrm{~Pb}+{ }^{208} \mathrm{~Pb}$ deepinelastic reaction. A large number of excited yrast and near-yrast states were identified on the top of the $11 / 2^{-}, \pi h_{11 / 2}^{-1}$ isomeric state. The level scheme is unambiguously identified up to spin $35 / 2$ at $6984 \mathrm{keV}$. The $17 / 2^{+}$state is of collective octupole character, while the other levels are understood to be associated with core excitations across the $N=126$ neutron shell gap. The ordering of these three-particle states is reproduced well by shell-model calculations. However, these calculations give a compressed energy spectrum at high spin. This spin dependent description of core-excited states is intrinsic to the nature of shell-model calculations with two-body matrix elements. This suggests that the structure of the predominantly three-particle states is more complex and multiple-core excitations need to be considered to remove the high-spin compression. In the future, the extension of the calculations to core-excited states using different modern effective interactions [33] could shed more light on the role of the interactions as well as that of the single-particle model space.

\section{Acknowledgements}

This work is supported by the Science and Technology Facilities Council (STFC), UK, the U.S. Department of Energy, Office of Science, Office of Nuclear Physics, under contract numbers DE-AC02-06CH11357, DE-FG02-94ER40834 and NSF grant PHY-140442, the Polish Ministry of Science and Higher Education under Contract number N-N202-263238. This research used resources of ANL's ATLAS facility, which is a DOE Office of Science user facility. The contributions of the Argonne National Laboratory technical staff are gratefully acknowledged.

\section{References}

[1] M. Goppert-Mayer, Phys. Rev. 74 (1948) 235.

[2] N. Bohr, Philos. Mag. Ser. 726 (1913) 1

[3] O. Echt, K. Sattler, E. Recknagel, Phys. Rev. Lett. 47 (1981) 1121.

[4] B. Fornal, et al., Phys. Rev. Lett. 87 (2001) 212501.

[5] N. Cieplicka, et al., Phys. Rev. C 86 (2012) 054322.

[6] C.J. Lister, J. Res. Natl. Inst. Stand. Technol. 105 (2000) 137.

[7] I.Y. Lee, Nucl. Phys. A 520 (1990) 641c.

[8] E. Wilson, et al., Acta Phys. Pol. B 44 (2013) 381.

[9] E. Wilson, et al., EPJ Web Conf. 66 (2014) 02110.

[10] E. Wilson, PhD thesis, University of Surrey, 2014, unpublished.

[11] F.G. Kondev, S. Lalkovski, Nucl. Data Sheets 112 (2011) 707.

[12] M. Rejmund, et al., Eur. Phys. J. A 8 (2000) 161.

[13] D. Eccleshall, M.J.L. Yates, Phys. Lett. 19 (1965) 301.

[14] M.J. Martin, Nucl. Data Sheets 63 (1991) 723.

[15] P.E. Haustein, et al., Nucl. Data Tables 10 (1972) 321.

[16] Zs. Podolyák, et al., J. Phys. Conf. Ser. 580 (2015) 012010.

[17] B.A. Brown, et al., OXBASH for Windows, MSU-NSCL report 1289, 2004.

[18] A. Hosaka, K.-I. Kubo, H. Toki, Nucl. Phys. A 444 (1985) 76.

[19] T.T.S. Kuo, G.H. Herling, Report No. 2258, US Naval Research Laboratory, 1971, unpublished.

[20] E.K. Warburton, B.A. Brown, Phys. Rev. C 43 (1991) 602.

[21] Zs. Podolyák, et al., Eur. Phys. J. A 42 (2009) 489.

[22] Zs. Podolyák, et al., Phys. Lett. B 672 (2009) 116.

[23] S.J. Steer, et al., Phys. Rev. C 78 (2008) 061302(R).

[24] S.J. Steer, et al., Phys. Rev. C 84 (2011) 044313.

[25] N. Al-Dahan, et al., Phys. Rev. C 80 (2009) 061302.

[26] G. Bertsch, et al., Nucl. Phys. A 284 (1977) 399.

[27] B.A. Brown, Phys. Rev. Lett. 85 (2000) 5300.

[28] A. Blazhev, et al., Phys. Rev. C 69 (2004) 064304.

[29] B. Fornal, et al., Phys. Rev. C 67 (2003) 034318.

[30] M. Rejmund, M. Schramm, K.H. Maier, Phys. Rev. C 59 (1999) 2520.

[31] R. Broda, et al., Eur. Phys. J. A 20 (2004) 145.

[32] M. Schramm, et al., Z. Phys. 344 (1992) 121.

[33] L. Coraggio, A. Covello, A. Gargano, N. Itaco, Phys. Rev. C 80 (2009) 021305(R). 